\title{
Dupilumab Maintains Long-Term Disease Control in Adults with Moderate-to-Severe Atopic Dermatitis as Measured by Well-Controlled Weeks: Results From the LIBERTY AD CHRONOS Clinical Trial
}

Jashin J. Wu • Lynda Spelman · Jerry L. Tan • Takafumi Etoh •

Haixin Zhang · Brad Shumel · Ana B. Rossi

Received: November 19, 2020 / Published online: January 28, 2021

(C) The Author(s) 2021

J. J. Wu (ه)

Dermatology Research and Education Foundation,

Irvine, CA, USA

e-mail: jashinwu@gmail.com

\section{Spelman}

Veracity Clinical Research and Probity Medical

Research, Brisbane, Australia

\section{J. L. Tan}

Windsor Clinical Research, Windsor, ON, Canada

T. Etoh

Tokyo Teishin Postal Services Agency Hospital,

Tokyo, Japan

H. Zhang · B. Shumel

Regeneron Pharmaceuticals, Inc., Tarrytown, NY, USA

A. B. Rossi

Sanofi Genzyme, Cambridge, MA, USA

\section{Key Summary Points}

In this analysis we aimed to assess disease control of moderate-to-severe atopic dermatitis (AD) in adults using the simple and pragmatic patient-reported outcome of well-controlled weeks

We found that $\mathrm{AD}$ patients receiving dupilumab plus topical corticosteroids (TCS) reported superior disease control during a 52-week treatment period, as assessed by patient-reported wellcontrolled weeks, compared to patients receiving placebo plus TCS

This simple patient-reported outcome measure offers additional confirmation that dupilumab treatment provides longterm disease control in $\mathrm{AD}$ patients, which has been previously established using other physician- and patient-reported efficacy assessments

We show that well-controlled weeks is a pragmatic and feasible measure of longterm disease control in patients with moderate-to-severe $\mathrm{AD}$ 


\section{DIGITAL FEATURES}

This article is published with digital features, including a summary slide, to facilitate understanding of the article. To view digital features for this article go to https://doi.org/10.6084/ m9.figshare.13554494.

Since its first availability in 2017, dupilumab has become an important treatment option for adult patients with moderate-to-severe atopic dermatitis (AD) that is not adequately controlled with topical therapy or when such therapies are not advisable. The rapid and sustained improvement in signs, symptoms, and quality of life and the long-term disease control achieved with dupilumab are demonstrated in a number of outcome measures evaluated during several randomized, phase 3 clinical trials [1]. Some of these outcome measures, however, are time consuming and involve calculations only used within a clinical trial setting. We aimed to assess disease control in adults with moderateto-severe $\mathrm{AD}$ who had been enrolled in the phase 3 CHRONOS study [2] using a simple and pragmatic patient-reported outcome: the number of well-controlled weeks [3-5] (Trial Registration: ClinicalTrials.gov Identifier: NCT02260986).

CHRONOS (NCT02260986) was a randomized, double-blind, placebo-controlled, phase 3 clinical trial conducted in patients with moderate-to-severe $\mathrm{AD}$ [2]. Patients were treated over a 1-year period with dupilumab $300 \mathrm{mg}$ weekly (qw) plus topical corticosteroids (TCS), dupilumab every 2 weeks ( $2 \mathrm{w}$ ) plus TCS, or placebo qw plus TCS. Patients had similar baseline disease characteristics across all treatment groups [2]. The median percentage of 'well-controlled' weeks over the 52-week treatment period was calculated from the percentage of patients in each treatment group responding positively to the question: 'In the last week was your AD well controlled?' Patients using rescue medication were considered non-responders, and missing values were handled using the last observation carried forward method.

As results for both dupilumab doses were similar, we present here only the results for the adult-approved dose (300 mg subcutaneous

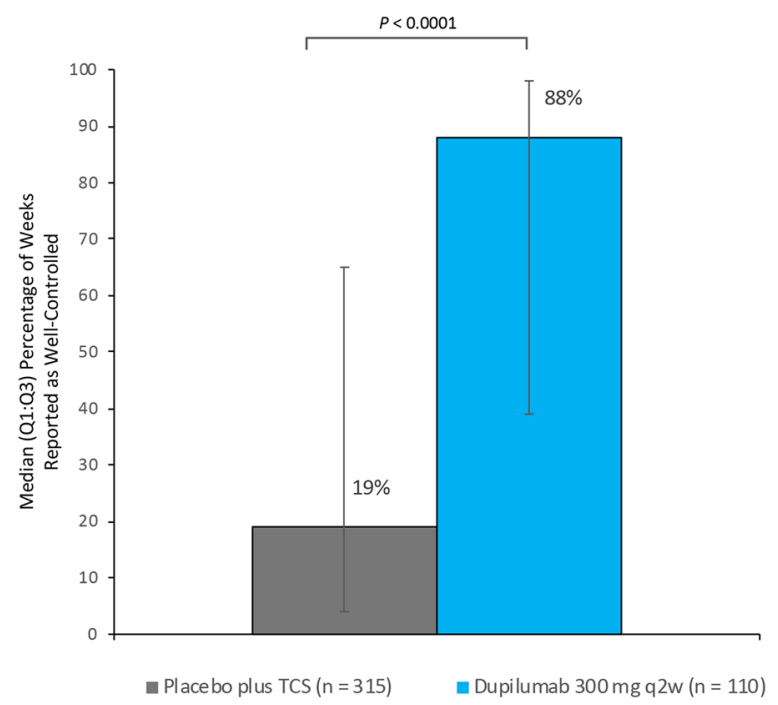

Fig. 1 Median percentage of well-controlled weeks (weeks $1-52$ ) by treatment group. $q 2 w$, every 2 weeks; TCS, topical corticosteroids. Patients using rescue medication were considered non-responders, and missing values were handled using the last observation carried forward method. Imputed patients: placebo $=94 / 315$; dupilumab $300 \mathrm{mg}$ $\mathrm{q} 2 \mathrm{w}=49 / 110$

injectable $\mathrm{q} 2 \mathrm{w}$ ). A total of 110 patients were randomized to dupilumab plus TCS and 315 patients to subcutaneous injectable placebo plus TCS. Over the course of 52 weeks, the median $(\mathrm{Q} 1: \mathrm{Q} 3$ interquartile range) percentage of weeks reported as well controlled was $88 \%$ (39:98) in the dupilumab plus TCS group compared to $19 \%$ (4:65) for patients treated with placebo plus TCS $(P<0.0001$, Fig. 1$)$.

Over the 52-week treatment period, patients receiving dupilumab plus TCS reported superior disease control as assessed by patient-reported well-controlled weeks. This simple outcome measure offers additional confirmation that treatment with dupilumab provides long-term disease control, which has been established using other physician- and patient-reported efficacy assessments.

While the burden of moderate-to-severe $\mathrm{AD}$ is not fully captured by clinical outcome measures, patient-reported assessments can provide a real-world assessment of disease status and treatment effectiveness from the patient's perspective. Given the chronic, relapsing nature of 
$\mathrm{AD}$, measuring long-term disease control is important. The Harmonizing Outcome Measures for Eczema (HOME) initiative includes long-term control as a key domain to be measured in all clinical trials of eczema $[4,5]$.

Although it is a simple and easy-to-understand concept, well-controlled weeks has not been fully validated as a patient-reported outcome in $\mathrm{AD}$ patients and needs further investigation. In contrast to other assessments, wellcontrolled weeks is a pragmatic and feasible measure of long-term disease control in patients with moderate-to-severe AD.

\section{ACKNOWLEDGEMENTS}

Funding. Research sponsored by Sanofi and Regeneron Pharmaceuticals, Inc. Sanofi and Regeneron Pharmaceuticals, Inc., also sponsored the journal's Rapid Service Fee.

Medical Writing and Editorial Assistance. Medical writing and editorial assistance provided by Lola MacRae, PhD, of Excerpta Medica, funded by Sanofi Genzyme and Regeneron Pharmaceuticals, Inc.

Authorship. All named authors meet the International Committee of Medical Journal Editors (ICMJE) criteria for authorship for this article, take responsibility for the integrity of the work as a whole, and have given their approval for this version to be published.

Disclosures. Jashin J. $\mathrm{Wu}$ is or has been an investigator, consultant, or speaker for AbbVie, Almirall, Amgen, Arcutis Biotherapeutics, Boehringer Ingelheim, Bristol-Myers Squibb, Celgene, Dermavant Sciences, Inc., Dermira Inc., Dr. Reddy's Laboratories, Eli Lilly, Janssen, LEO Pharma, Novartis, Regeneron Pharmaceuticals, Inc., Sanofi Genzyme, Sun Pharmaceutical, UCB, and Valeant Pharmaceuticals North America LLC. Lynda Spelman has served on advisory boards for AbbVie, Eli Lilly, Galderma, Janssen, and Novartis and has served as an investigator for AbbVie, Amgen, Anacor, Ascend Biopharmaceuticals, Astellas Pharma US,
Australian Wool Innovation Limited, Blaze Bioscience, Boehringer Ingelheim, Bristol-Myers Squibb, Botanix, Celgene, Dermira, Eli Lilly, Galderma, Genentech, GlaxoSmithKline, Janssen, Kythera, LEO Pharma, Mayne Sun Pharma, Merck, Novartis, Pfizer, Phosphagenics, Regeneron Pharmaceuticals, Inc., Sanofi, SHR, Sun Pharmaceutical, Trius, UCB, and Zai Lab. Jerry L. Tan has been an advisor and investigator for Regeneron Pharmaceuticals, Inc. Takafumi Etoh received honoraria for lectures at Kyowa Hakko Kirin and Maruho Medical, Inc. Haixin Zhang and Brad Shumel are employees and shareholders of Regeneron Pharmaceuticals, Inc. Ana B. Rossi is an employee and may hold stock and/ or stock options in Sanofi.

Compliance with Ethics Guidelines. This study was conducted in accordance with the Declaration of Helsinki, International Conference on Harmonisation Good Clinical Practice guidelines (version R1), and applicable regulatory requirements. All patients provided signed written informed consent. The protocol and all relevant study forms were approved by all relevant institutional review boards and an independent ethics committee. An independent data monitoring committee monitored patient safety.

Open Access. This article is licensed under a Creative Commons Attribution-NonCommercial 4.0 International License, which permits any non-commercial use, sharing, adaptation, distribution and reproduction in any medium or format, as long as you give appropriate credit to the original author(s) and the source, provide a link to the Creative Commons licence, and indicate if changes were made. The images or other third party material in this article are included in the article's Creative Commons licence, unless indicated otherwise in a credit line to the material. If material is not included in the article's Creative Commons licence and your intended use is not permitted by statutory regulation or exceeds the permitted use, you will need to obtain permission directly from the copyright holder. To view a copy of this licence, visit http://creativecommons.org/licenses/by$\mathrm{nc} / 4.0 /$. 


\section{REFERENCES}

1. Sastre J, Dávila I. Dupilumab: a new paradigm for the treatment of allergic diseases. J Investig Allergol Clin Immunol. 2018;28(3):139-50.

2. Blauvelt A, de Bruin-Weller M, Gooderham M, et al. Long-term management of moderate-to-severe atopic dermatitis with dupilumab and concomitant topical corticosteroids (LIBERTY AD CHRONOS): a 1-year, randomised, double-blinded, placebo-controlled, phase 3 trial. Lancet. 2017;389(10086):2287-303.

3. Langan SM, Stuart B, Bradshaw L, et al. Measuring long-term disease control in patients with atopic dermatitis: a validation study of well-controlled weeks. J Allergy Clin Immunol. 2017;140(6):1580-6.

4. Barbarot S, Rogers NK, Abuabara K, et al. Strategies used for measuring long-term control in atopic dermatitis trials: a systematic review. J Am Acad Dermatol. 2016;75(5):1038-44.

5. Howells L, Thomas KS, Sears AV, et al. Defining and measuring "eczema control": an international qualitative study to explore the views of those living with and treating atopic eczema. J Eur Acad Dermatol Venereol. 2019;33(6):1124-32. 